\title{
Correction to: Community detection and unveiling of hierarchy in networks: a density-based clustering approach
}

\author{
Zineb Felfli', Roy George, Khalil Shujaee and Mohamed Kerwat
}

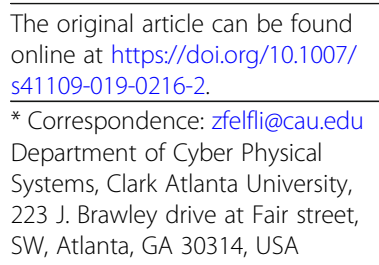

* Correspondence: zfelfli@cau.edu Department of Cyber Physical Systems, Clark Atlanta University, $223 \mathrm{~J}$. Brawley drive at Fair street, SW, Atlanta, GA 30314, USA

\author{
Correction to: Appl Netw Sci (2019) 4:85 \\ https://doi.org/10.1007/s41109-019-0216-2
}

Following the publication of the original article (Felfli et al. 2019), multiple errors were identified in the sections and Figure 1.

The changes have been highlighted in bold typeface.

Abstract:

We experimentally evaluate a statistical mechanics approach, the Correlation Density Rank, that uses a normalized Gaussian function which captures the impact of a node within its neighborhood and leads to a density-ranking of nodes by considering the distance between nodes as punishment.

The technique uses hill-climbing procedure to determine the density attractors and identify the unique parent (leader) of each member as well as the group leader.

The method is exhaustively tested using synthetic networks generated by the LFR benchmarking algorithm for network sizes between 500 and 30000 nodes and mixing parameter between 0.1 and 0.9 .

Introduction:

The approach is based on previous work [25][26], which evaluated a small synthetic network.

In the present paper, we use larger networks generated by the LFR benchmark in an attempt to establish the validity and utility of the approach and identify its limitations.

The approach assumes a Gaussian density distribution which is constructed so as to unveil the relative importance (influence) of the various nodes (members) of the network, allowing for the identification of the immediate leader of every member and hence the ranking of all members including the emergence of the group leader.

Section 4 concludes the paper, evaluates the performance of the algorithm and addresses its limitations. 


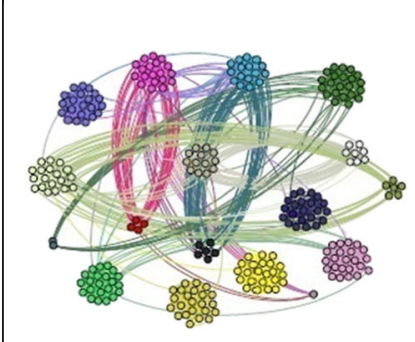

(a)

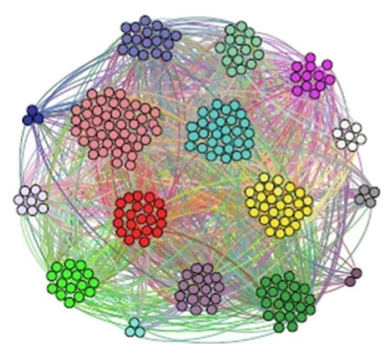

(b)

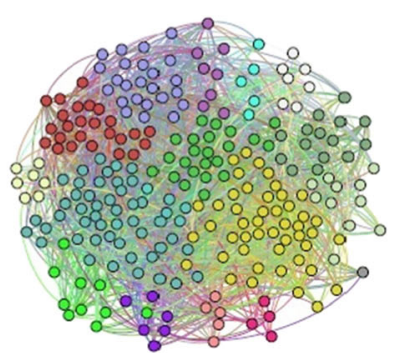

(c)

Fig. 1 Results of the clustering algorithm corresponding to LFR graphs with $N=250$ and (a) $\mu=0.1$, (b) $\mu=$ 0.3 and (c) $\mu=0.5$

Approach:

The distance matrix is mapped into a Gaussian kernel matrix [26] which is a nonlinear Euclidean distance (a radial basis function (RBF) kernel and commonly used in Support Vector Machine classification).

The density function first introduced in [25] considers the distance between nodes as punishment and captures the impact of a node within its neighborhood.

The normalized Gauss influence function takes the form

To illustrate this outcome, we show in Figure 1 the results of the clustering method for LFR- benchmark graphs with $N=250$ and mixing parameter, $\mu=0.1,0.3$ and 0.5 .

Results:

The dependence of the average degree on the network size and, in the case of scale free networks, to the degree distribution exponent is quite loose [27].

The benchmarking procedure allowed us to assess the quality of the present formulation. Work on extending the methodology so that overlapping communities are taken into account, is in progress.

Discussion and Conclusions:

The benchmarking process allowed us to assess the performance of the approach.

References:

[26] Z. Bahrami Bidoni and R. George, "Network service quality rank: a network selection algorithm for heterogeneous wireless networks," ACM/IEEE Symposium on Architectures for Networking and Communications Systems (ANCS) (pp. 239240). IEEE.

[27] G. K. Orman, V. Labatut and H. Cherifi, "Towards Realistic Artificial Benchmark for Community Detection Algorithms Evaluation," International Journal of Web Based Communities 9 (3), 349-370, 2013

[28] Pablo Meyer et al, Network topology and parameter estimation: from experimental design methods to gene regulatory network kinetics using a community-based approach, BMC Systems Biology 2014, 8:13 Towards Realistic Artificial Benchmark for Community Detection Algorithms Evaluation

Published online: 30 July 2020

Reference

Felfli Z et al (2019) Community detection and unveiling of hierarchy in networks: a density-based clustering approach. Appl Netw Sci 4:85. https://doi.org/10.1007/s41109-019-0216-2 